\title{
Identification of Nuclear Localization Signals in the Human Homeoprotein, MSX1
}

\begin{tabular}{|c|c|}
\hline Journal: & Biochemistry and Cell Biology \\
\hline Manuscript ID & bcb-2017-0263.R1 \\
\hline Manuscript Type: & Note \\
\hline Date Submitted by the Author: & 31-Oct-2017 \\
\hline Complete List of Authors: & $\begin{array}{l}\text { Shibata, Akio; Aichi Gakuin Daigaku Shigakubu Daigakuin Shigaku } \\
\text { Kenkyuka } \\
\text { Machida, Junichiro; Toyota Kinen Byoin, Department of Oral and } \\
\text { Maxillofacial Surgery } \\
\text { Yamaguchi, Seishi; Aichi Gakuin Daigaku Shigakubu Daigakuin Shigaku } \\
\text { Kenkyuka } \\
\text { Kimura, Masashi; Ogaki Shimin Byoin } \\
\text { Tatematsu, Tadashi; Aichi Gakuin Daigaku Shigakubu Daigakuin Shigaku } \\
\text { Kenkyuka } \\
\text { Miyachi, Hitoshi; Aichi Gakuin Daigaku Shigakubu Daigakuin Shigaku } \\
\text { Kenkyuka } \\
\text { Nakayama, Atsuo; Aichi Human Service Center } \\
\text { Shimozato, Kazuo; Aichi Gakuin Daigaku Shigakubu Daigakuin Shigaku } \\
\text { Kenkyuka } \\
\text { TOKITA, Yoshihito; Aichi Human Service Center }\end{array}$ \\
\hline $\begin{array}{r}\text { Is the invited manuscript for } \\
\text { consideration in a Special } \\
\text { Issue? : }\end{array}$ & N/A \\
\hline Keyword: & homeodomain (HD), nuclear localization signal (NLS), Msh homeobox 1 \\
\hline
\end{tabular}


1 Identification of Nuclear Localization Signals in the Human Homeoprotein, MSX1

2

3 Akio Shibata $^{1,2}$, Junichiro Machida ${ }^{1,3}$, Seishi Yamaguchi ${ }^{1,4}$, Masashi Kimura ${ }^{1,5}$, Tadashi

4 Tatematsu $^{1,2}$, Hitoshi Miyachi ${ }^{1}$, Atsuo Nakayama ${ }^{6}$, Kazuo Shimozato $^{1}$, Yoshihito Tokita ${ }^{2}$

61 Department of Maxillofacial Surgery, Aichi-Gakuin University School of Dentistry, Nagoya,

7 Japan, 2 Department of Perinatology, Institute for Developmental Research, Aichi Human

8 Service Center, Kasugai, Japan, 3 Department of Oral and Maxillofacial Surgery, Toyota

9 Memorial Hospital, Toyota, Japan, 4 Department of Dentistry and Oral Surgery, Aichi

10 Children's Health and Medical Center, Obu, Japan, 5 Department of Oral and Maxillofacial

11 Surgery, Ogaki Municipal Hospital, Ogaki, Japan, 6 Department of Embryology, Institute for

12 Developmental Research, Aichi Human Service Center, Kasugai, Japan

13

14 *Correspondence: Y. Tokita, Department of Perinatology, Institute for Developmental

15 Research, Aichi-Human Service Center, Kamiya, 713-8, Kasugai, Aichi 480-0392, Japan

16 Tel: $+81568880811 \quad$ Fax: +81568880829

17 E-mail: tokita@inst-hsc.jp 


\section{Abstract}

20 MSX1 is one of the homeoproteins with the homeodomain (HD) sequence, which regulates

21 proliferation and differentiation of mesenchymal cells. In this study, we investigated the

22 nuclear localization signal (NLS) in the MSX1 HD by deletion and amino acid substitution

23 analyses. The web-based tool NLStradamus predicted two putative basic motifs in the N- and

24 C-termini of the MSX1 HD. Green fluorescent protein (GFP) chimera studies revealed that

25 NLS1 $\left({ }_{161}\right.$ RKHKTNRKPR $\left._{170}\right)$ and NLS2 $\left({ }_{216} \mathrm{NRRAKAKR}_{223}\right)$ were independently

26 insufficient for robust nuclear localization. However, they can work cooperatively to promote

27 nuclear localization of MSX1, as was shown by the two tandem NLS motifs partially

28 restoring functional NLS, leading to a significant nuclear accumulation of the GFP chimera.

29 These results demonstrate a unique NLS motif in MSX1, which consists of an essential single

30 core motif in helix-I, with weak potency, and an auxiliary subdomain in helix-III, which alone

31 does not have nuclear localization potency. Additionally, other peptide sequences, other than

32 predicted two motifs in the spacer, may be necessary for complete nuclear localization in

33 MSX1 HD.

34 Keywords; MSX1, homeodomain (HD), nuclear localization signal (NLS) 


\section{Introduction}

37 Homeoproteins are transcription factors that regulate cell proliferation and differentiation

38 Ladam and Sagerstrom 2014. These proteins contain a highly conserved polypeptide, termed

39 the homeodomain (HD). This domain enables them to interact with target DNA sequences,

40 and also several other proteins, to regulate various biological processes Zhang et al. 1996; Ye

et al. 2011; Hovde et al. 2001. Homo- and heterodimeric protein-protein interactions are

considered particularly important for regulating the actions of HD-containing transcriptional

regulatory proteins, including Msx homeoprotein families Zhang et al. 1996; Zhang et al.

44 1997. The expression of MSX1 is tightly controlled, both spatially and temporally, during

45 development as this gene regulates the proliferation and differentiation of mesenchymal cells

Zhang et al. 1997. Msx genes play a critical role in craniofacial development, and $M s x 1^{-/-}$

47 mouse embryos develop with a complete secondary cleft palate, as well as tooth agenesis. The

48 MSX1 protein harbors a HD sequence (amino acids 167-228), which interacts with target

49 proteins, such as octamer-binding transcription factor 1 (OCT1), TATA-binding protein (TBP),

50 distal-less homeobox (DLX), and enhancer of zeste 2 (EZH2) Zhang et al. 1997; Rave-Harel

51 et al. 2005.

52 Nuclear proteins translocate into the nucleus through nuclear pore complexes (NPCs) 
53 Grunwald and Singer 2012. Small proteins can translocate through NPCs by passive diffusion,

54 whereas active translocation is essential for larger proteins Lange et al. 2007. However, even

55 small proteins, such as histones, promote translocation by an active process Breeuwer and

56 Goldfarb 1990. These active processes are mediated by a nuclear localization signal (NLS)

57 composed of basic amino acid residues Gorlich and Mattaj 1996. Classical monopartite or

58 bipartite NLS sequences are recognized by the importin $\alpha$ and $\beta$ heterodimer complex, and the

59 proteins containing these moieties are translocated into the nucleus via an energy-dependent

60 process Gorlich and Mattaj 1996; Lee et al. 2006. In classical NLS, importin $\alpha$ recognizes the

61 NLS through a specific binding site and importin $\beta$ mediates translocation of the protein

62 through the NPCs. Furthermore, previous studies indicated that several homeoproteins, with

63 unique NLS, required a different manner of interaction with importin $\alpha$ and/or $\beta$ Ye et al. 2011.

64 We hypothesize that MSX1 (33 kDa) may undergo nuclear localization in a similar manner to

65 histones, and predict that functional NLS sites would be embedded in this protein.

66 Several studies have suggested that the HD of MSX1 contains NLS sequences Wang et al.

67 2011; Kimura et al. 2014. Although the HD is a highly conserved region, amino acid sequence

68 divergence is observed in the NLS regions of homeoproteins Ye et al. 2011. In this study, we

69 investigated the intracellular distribution of several MSX1 mutant proteins with deletions or 
70 amino acid substitutions in cultured HEK 293 cells to identify the functional nuclear

71 localization motifs of MSX1. We identified two isolated basic motifs, located within the

72 N-terminus and C-terminus of the HD, and reveal that both the motifs are crucial for nuclear

73 localization of MSX1.

74

\section{$75 \quad$ Material and methods}

\section{Plasmid construction}

77 The cDNA encoding the MSX1 protein was amplified from human genomic DNA by PCR as

78 previously described Kamamoto et al. 2011. This cDNA fragment was then cloned into the

79 pcDNA3 vector (Invitrogen, Carlsbad, CA), harboring the FLAG epitope-tag at the 5' end of

80 the coding region. Site-directed mutagenesis and deletions were carried out using the PCR

81 megaprimer method and/or inverse PCR method, respectively (Supplemental table 1)

82 Kamamoto et al. 2011; Ochman et al. 1988. For detection of HD deletion mutants, we used

83 EGFP as the FLAG fusion proteins were not detected in the transfected cells, likely due to

84 protein instability (data not shown). To obtain an EGFP fusion protein, the fragments were

85 digested using $B g l \mathrm{II}$ and SalI and ligated into the pEGFP-C1 vector (Clontech, Mountain

86 View, CA). All constructs were verified by DNA sequencing. 

Immunolocalization of various MSX1 mutants

89 Human embryonic kidney 293 (HEK 293; ATCC \#CRL-1573) cells were cultured in

90 Dulbecco's modified Eagle's medium (DMEM) supplemented with 10\% (v/v) fetal bovine

91 serum at $37^{\circ} \mathrm{C}$ in an atmosphere of $5 \% \mathrm{CO}_{2}$. For transfection and immunostaining, cells were

92 plated onto 12-well poly-L-lysine-coated cover glass (12 $\mathrm{mm}$ in diameter; Matsunami, Osaka,

93 Japan) at a density of $10^{4}$ cells/well. After 2-3 days, transient transfections were carried out

94 using Lipofectamine 2000 (Invitrogen). $24 \mathrm{~h}$ after transfection, the cells were fixed with

$953.75 \%(\mathrm{w} / \mathrm{v})$ paraformaldehyde/phosphate buffered saline (PBS) and permeabilized with $0.2 \%$

96 (v/v) Triton X-100/PBS and then incubated with the mouse anti-FLAG monoclonal antibody

97 (1:1000 dilution; Sigma-Aldrich, St. Louis, MO) or rabbit anti-GFP antibody (1:1000

98 dilution; Invitrogen), overnight at $4^{\circ} \mathrm{C}$. The cells were subsequently incubated for 60 min at

99 room temperature with either Cy3-conjugated goat anti-mouse antibody (1:1000 dilution;

100 Jackson ImmunoResearch Laboratories, West Grove, PA) or Alexa Fluor ${ }^{\circledR} 488$ donkey

101 anti-rabbit antibody (1:1000 dilution; Invitrogen). 4', 6-Diamidino-2-phenylindole (DAPI; 1

$102 \mu \mathrm{g} / \mathrm{mL}$ ) was used to detect nuclei.

103 


\section{Microscopy and statistical analysis}

105 After washing three times with PBS, cells were visualized under an Olympus BH-2

106 microscope (Olympus, Tokyo, Japan). The images were captured using Photoshop Elements

10711 software (Adobe, San Jose, CA) and analyzed using ImageJ Schneider et al. 2012 to

108 calculate the quantifying cellular distribution of MSX1 mutant proteins as previously

109 described, with minor modifications Gueroussov et al. 2010. Briefly, cells were analyzed by

110 measuring the average fluorescence of the fractions, which is manually separated nuclear,

111 cytoplasmic, and intracellular distribution, using the following equation:

$$
\% \text { of Nuclear fluorescence }=\left(\frac{A_{N u c} \times\left(F_{N u c}-F_{B a c k}\right)}{A_{T o l} \times\left(F_{T o l}-F_{B a c k}\right)}\right) \times 100
$$

$112 \quad \mathrm{~A}_{\mathrm{Nuc}}$; Area of the nucleus

$113 \quad \mathrm{~A}_{\mathrm{Tol}}$; Area of the whole cell

$114 \quad \mathrm{~F}_{\mathrm{Nuc}}$; Average fluorescence of the nuclear fraction

$115 \quad \mathrm{~F}_{\text {Tol }}$; Average fluorescence of the whole cell fraction

$116 \quad \mathrm{~F}_{\mathrm{Back}} ;$ Average fluorescence of an untransfected cell

117 The percentage of nuclear fluorescence represents the mean \pm standard error of the mean

118 (SEM). At least 50 cells were counted in five randomly selected fields in two or three

119 independent experiments. Statistical analysis was performed using the Welch's $t$-test. A 
$120 P$-value of $<0.001$ was considered a statistically significant difference between experimental

121 groups.

122

123 Preparation of Nuclear and Cytosolic Extracts

124 Nuclear and cytosolic extracts were separated as previously described, with minor

125 modifications Garcia-Yague et al. 2013. Briefly, the cell pellet was resuspended in three pellet

126 volumes of cold buffer A (20 mM HEPES, pH 7.0, 0.15 mM EDTA, 0.015 mM EGTA, 10

$127 \mathrm{mM} \mathrm{KCl}, 1 \%$ Nonidet P-40, $1 \mathrm{mM}$ phenylmethylsulfonyl fluoride, $20 \mathrm{mM} \mathrm{NaF}, 1 \mathrm{mM}$

128 sodium pyrophosphate, $1 \mathrm{mM}$ sodium orthovanadate, and $1 \mu \mathrm{g} / \mathrm{mL}$ leupeptin) and incubated

129 on ice for $30 \mathrm{~min}$. Then the homogenate was centrifuged at $500 \times g$ for $5 \mathrm{~min}$. The

130 supernatants were taken as the cytosolic fraction. The nuclear pellet was resuspended in five

131 volumes of cold buffer B (10 mM HEPES, pH 8.0, 0.1 mM EDTA, $0.1 \mathrm{mM} \mathrm{NaCl,} \mathrm{25 \%}$

132 glycerol, $1 \mathrm{mM}$ phenylmethylsulfonyl fluoride, $20 \mathrm{mM} \mathrm{NaF}, 1 \mathrm{mM}$ sodium pyrophosphate, 1

$133 \mathrm{mM}$ sodium orthovanadate, and $1 \mu \mathrm{g} / \mathrm{mL}$ leupeptin). After centrifugation, in the same

134 conditions outlined above, the nuclei were resuspended in loading buffer containing $0.5 \%$

135 SDS.

136 


\section{Western blotting}

138 The cytosolic and nuclear fractions were resolved by western blotting as previously described,

139 with minor modifications Tokita et al. 2001. Briefly, HEK 293 transfectants were extracted

140 with lysis buffer (1\% Triton X-100, 1 mM EDTA in Tris-buffered saline; TBS) and these

141 lysates were run on an SDS-PAGE (12\% gel) and transferred to Immobilon-P membranes

142 (Millipore Corporation, Bedford, MA). The membranes were blocked with 1\% bovine serum

143 albumin (BSA) in TBS for $1 \mathrm{~h}$ at room temperature and then probed with an anti-GFP

144 antibody (1:2000, Invitrogen). Control experiments were performed using rabbit anti-histone

145 H3 K27me3 antibodies (1:3000, Millipore), and rabbit anti-alpha tubulin antibodies (1:1000,

146 Genetex, Irvine, CA). The signals on the blots were detected with horseradish

147 peroxidase-conjugated secondary antibodies (1:400, Vector Laboratories, Burlingame, CA)

148 and Western Lightning ECL Pro (PerkinElmer, Winter Street Waltham, MA).

149

$150 \quad$ Results

\section{Prediction of MSX1 nuclear localization signal}

152 The molecular weight of MSX1 protein is $\sim 31 \mathrm{kDa}$, which means it can enter the nucleus by

153 passive diffusion. However, several reports have suggested that the nuclear localization of 

MSX1 is dependent on its $\mathrm{HD}_{2}$ since wild-type MSX1 and its entire HD was exclusively

155 localized in the nucleus, and HD-deleted MSX1 $(\Delta \mathrm{HD})$ was only diffusely localized in the

156 cytoplasm (Fig. 1) Wang et al. 2011; Kimura et al. 2014. These findings indicate that the HD

157 is indispensable to nuclear localization and that one or more functional NLS sites are present

158 in the MSX1 HD. NLS motifs were predicted using a web-based tool, NLStradamus Nguyen

159 et al. 2009. We identified two putative NLS regions containing multiple positively charged

160 residues at amino acids 161-170 and 216-223 within the HD. We designated them NLS1

$161 \quad\left({ }_{161}\right.$ RKHKTNRKPR $\left._{170}\right)$ and NLS2 (216NRRAKAKR 223$)$ (Fig. 1A).

162

\section{Mapping of NLS sites in MSX1}

164 We first constructed several EGFP-fused proteins of the MSX1 HD, both wild-type and

165 deletion mutants. Whereas a significant reduction in the nuclear fluorescence signal intensity

166 was observed for the FLAG- $\triangle \mathrm{HD}$ when compared with FLAG-Wild type (Fig. 1C),

167 FLAG-HD and EGFP-HD had similar nuclear fluorescence intensity to FLAG-Wild MSX1

168 (Fig. 1, 2). These results indicate that the nuclear localization of FLAG-HD, EGFP-HD, and

169 FLAG-Wild MSX1 are partially directed by active transport, because the nuclear fluorescence 
171 enter the nucleus by passive diffusion Pan et al. 2004; Sato et al. 2013; Hatayama et al. 2008.

172 We then constructed EGFP fusion proteins with HDN' ${ }_{161-205}$ and HDC' ${ }_{205-228}$ (Fig. 2A). While

173 the immunolocalization of the NLS1-containing fusion protein (EGFP-HDN') was detected

174 predominantly in the nucleus, the NLS2-containing chimeric protein (EGFP-HDC') was

175 detected in the cytoplasm (Fig. 2B, C). Next, to determine whether the NLS1 or NLS2 were

176 required for nuclear localization of MSX1, we examined the subcellular distribution of the

177 EGFP fusion products of the HD mutants without NLS1 or NLS2, respectively (Fig. 2A). As

178 shown in Fig. 2B and C, the subcellular localization of EGFP- $\triangle$ NLS1 was similar to that of

179 EGFP, whereas EGFP-ANLS2 mutant HD was observed predominantly in the nucleus. As

180 shown in Fig. 2C, there are no significant differences between EGFP- $\triangle$ NLS1, EGFP-HDC',

181 and EGFP.

182

183 Cooperative effect of the isolated NLS1 and NLS2 motifs

184 To determine whether NLS1 alone is enough to direct the EGFP to the nuclei independently,

185 we analyzed the immunolocalization of the EGFP-NLS1, and two tandem NLS

186 (EGFP-NLS1+2; Fig. 3A). EGFP-NLS1+2 chimeric protein was predominantly localized in

187 the nucleus, whereas EGFP-NLS1 was observed in the cytoplasm, indicating that NLS2 is one 
188 of the crucial signals for nuclear localization mediated by NLS1 (Fig. 3B, C). Interestingly,

189 there was equal nuclear fluorescence between EGFP-NLS1+2, EGFP- $\Delta$ NLS2, and

190 EGFP-HDN'. However, the nuclear fluorescence was significantly different between

191 EGFP-NLS1+2 and EGFP-HD (Fig. 3C). To validate the NLS potency of these two tandem

192 peptides biochemically, we performed western blotting analysis. While almost all EGFP

193 protein is detected in the cytosolic fraction, significant amounts of EGFP-NLS1+2 and

194 EGFP-HD proteins are detected in the nuclear fraction (Fig. 3D). These results demonstrated

195 that the two tandem NLS peptides promote nuclear localization.

196 Finally, to characterize the two tandem NLS peptides precisely, we performed site-directed

197 mutagenesis in NLS1+2 to generate mutants: EGFP-mNLS1N', EGFP-mNLS1C',

198 EGFP-mNLS1, EGFP-mNLS2, and EGFP-mNLS1+2, which have mutated the basic amino

199 acid residues to an Ala residue (Fig. 4A). Whereas the immunolocalization of

200 EGFP-mNLS1C' was observed mostly in the nucleus, the immunolocalization of both

201 EGFP-mNLS1N', EGFP-mNLS1, EGFP-mNLS2, and EGFP-mNLS1+2 was observed mostly

202 in the cytoplasm (Fig. 4B, C). There was no significant difference between EGFP-mNLS1N',

203 EGFP-mNLS1, EGFP-mNLS1+2, and EGFP. Interestingly, considerable nuclear localization

204 of EGFP- $\triangle$ NLS2 was observed (Fig. 2C), whereas only a small amount of nuclear 
localization of EGFP-mNLS2 was observed (Fig. 4C).

206

207 Discussion

208 Generally, the folding structures of HDs are highly conserved and consist of three alpha

209 helices: helix-I, -II, and, -III. Helix-II and -III form a helix-turn-helix motif, and helix-III

210 recognizes specific DNA motifs Hovde et al. 2001; Damante et al. 1994. NLS sites in the HD

211 can be classified according to previous studies and our similar observations (Table 1).

212 Monopartite NLS sites within the helix-I or helix-III have been identified in some

213 homeoproteins such as Tst-1/Oct6, PDX-1, and PITX2, which are very similar to the NLS

214 sequence of SV40 T-antigen Sock et al. 1996; Kozlowski and Walter 2000; Moede et al. 1999.

215 Bipartite NLSs have a spacer between two interdependent basic amino acid residue clusters in

216 the N-terminal region of the HD, in close proximity to helix I. It has been reported that the

217 spacer peptide sequence of DLX3 is not necessary for nuclear localization Robbins et al.

218 1991; Bryan and Morasso 2000. Two NLSs are observed in Nanog, Nkx2.2 and TTF-1, and

219 are characterized by two independent NLS motifs in helix-I and helix-III, which can

220 cooperate for complete nuclear localization Do et al. 2007; Hessabi et al. 2000;

221 Christophe-Hobertus et al. 1999. However, these studies do not address the functions of the 
222 spacer sequence between the two NLS motifs.

223 In our study, we reveal that MSX1 contains a characteristic tandem NLS, which are two basic

224 motifs located at the N-terminus of the HD and in helix-III. Although each of the predicted

225 NLSs, NLS1 $\left({ }_{161}\right.$ RKHKTNRKPR $\left._{170}\right)$ and NLS2 $\left({ }_{216} \mathrm{NRRAKAKR}_{223}\right)$, is insufficient for

226 nuclear localization alone, when they cooperate there is considerable nuclear localization. We

227 demonstrate for the first time that a tandem arrangement of two isolated NLS motifs within a

228 HD can direct a foreign protein to the nucleus. Furthermore, we conclude that the peptide

229 sequence containing three basic amino acid residues in the N-terminus of NLS1 (161 $\left.\underline{\mathrm{RK}} \underline{K}_{164}\right)$

230 is pivotal for the nuclear localization of human MSX1 since EGFP- $\Delta$ NLS1 and

231 EGFP-mNLS1N' were mostly detected in the cytoplasm and EGFP-mNLS1C' in the nucleus.

232 However, the GFP chimera with NLS1 alone has only partial potency. These results clearly

233 suggest that the N-terminus of NLS1 is insufficient alone, but plays a necessary part in the

234 NLS.

235 NLS2 cannot function by itself; nevertheless, it plays an important role in the NLS mediated

236 by the N-terminal of NLS1. This is because NLS2 greatly recovers nuclear localization, and

237 the alanine substitutions of NLS2 seriously affect the nuclear localization of the tandem NLS

238 chimera. EGFP- $\triangle$ NLS2 immunolocalization was detected mostly in the nucleus and EGFP 
239 NLS1+2 fusion protein cannot fully recover the nuclear localization potency of the native

240 NLS. These observations imply that the spacer peptide sequence of NLS1+2 is necessary for

241 nuclear localization. The spacer sequence, for instance ${ }_{183} \mathrm{RKFRQK}_{188}$, close to NLS1, may be

242 important for nuclear localization as EGFP-HD'N showed predominant nuclear localization

243 compared with EGFP-NLS1.

244 It has been demonstrated that proteins with two NLSs require a strict tertiary structure to

245 function Ye et al. 2011; Wong et al. 2014. Thus, we predict that some alteration in the NLS

246 structure of the NLS1+2 chimeric GFP, because some proteins with two NLSs, such as

247 Nkx2.2, bind importin $\beta$ directly after folding, whereas monopartite and bipartite NLSs can be

248 directly recognized either by importin $\alpha$ or importin $\beta$ Ye et al. 2011. In the two NLSs of

249 TTF-1, the unique tripeptide Trp-Phe-Gln (WFQ), near the basic amino acid cluster in the

250 C-terminus of HD, is highly conserved across species. An alanine replacement in this

251 tripeptide affects the nuclear localization of the GFP chimeric protein Christophe-Hobertus et

252 al. 1999. Furthermore, a nonstop mutation prevents the nuclear localization of MSX1 with

253 intact HD due to an additional 48 amino acid residue at the C-terminus, which would likely

254 alter the protein's structure Wong et al. 2014. Based on the findings of these reports and our

255 study, we speculate that the three-dimensional structure is also crucial for full potency for the 
256 two NLSs in human MSX1, and that the N-terminal of HD certainly would include other

257 crucial peptide sequences which can improve the nuclear localization potency of NLS1.

258 Finally, we demonstrate that two isolated basic motifs within the HD of MSX1 play pivotal

259 roles in nuclear localization. Furthermore, our observations suggest that the NLS sequence

260 can significantly promote nuclear localization of small proteins, such as MSX1. Though it has

261 been previously demonstrated that the nuclear transports of several homeoproteins are

262 mediated by importin families Ye et al. 2011, the molecular mechanism of nuclear transport of

263 MSX1 was not known. Further studies are required to elucidate the precise molecular

264 mechanisms of nuclear transport mediated by the two NLS sites in MSX1.

265 Our study demonstrates a unique NLS motif in MSX1, which consists of an essential single

266 core motif at the region close to helix-I with weak potency, and an auxiliary subdomain in the

267 helix-III that alone does not contribute to nuclear localization potency.

268

269 Acknowledgment

270 This study was supported in part by grants from the Institute for Developmental Research,

271 Aichi-Human Service Center (YT), and from the Aichi-Gakuin University, School of

272 Dentistry (KS). The authors declare that they have no competing financial interests. 


\section{References}

276 Breeuwer, M., and Goldfarb, D.S. 1990. Facilitated nuclear transport of histone H1 and other

277 small nucleophilic proteins. Cell 60(6): 999-1008.

278 Bryan, J.T., and Morasso, M.I. 2000. The Dlx3 protein harbors basic residues required for

279 nuclear localization, transcriptional activity and binding to Msx1. J. Cell Sci. 113 ( Pt 22):

$280 \quad 4013-4023$.

281 Christophe-Hobertus, C., Duquesne, V., Pichon, B., Roger, P.P., and Christophe, D. 1999.

282 Critical residues of the homeodomain involved in contacting DNA bases also specify the

283 nuclear accumulation of thyroid transcription factor-1. Eur. J. Biochem. 265(1): 491-497.

284 Damante, G., Fabbro, D., Pellizzari, L., Civitareale, D., Guazzi, S., Polycarpou-Schwartz, M.,

285 Cauci, S., Quadrifoglio, F., Formisano, S., and Di Lauro, R. 1994. Sequence-specific DNA

286 recognition by the thyroid transcription factor-1 homeodomain. Nucleic Acids Res. 22(15):

$287 \quad 3075-3083$.

288 Do, H.J., Lim, H.Y., Kim, J.H., Song, H., Chung, H.M., and Kim, J.H. 2007. An intact

289 homeobox domain is required for complete nuclear localization of human Nanog. Biochem.

290 Biophys. Res. Commun. 353(3): 770-775. doi: 10.1016/j.bbrc.2006.12.100.

291 Garcia-Yague, A.J., Rada, P., Rojo, A.I., Lastres-Becker, I., and Cuadrado, A. 2013. Nuclear 
292 import and export signals control the subcellular localization of Nurrl protein in response 293 to oxidative stress. J. Biol. Chem. 288(8): 5506-5517. doi: 10.1074/jbc.M112.439190.

294 Gorlich, D., and Mattaj, I.W. 1996. Nucleocytoplasmic transport. Science 271(5255): $295 \quad 1513-1518$.

296 Grunwald, D., and Singer, R.H. 2012. Multiscale dynamics in nucleocytoplasmic transport. 297 Curr. Opin. Cell Biol. 24(1): 100-106. doi: 10.1016/j.ceb.2011.11.011.

298 Gueroussov, S., Tarnawsky, S.P., Cui, X.A., Mahadevan, K., and Palazzo, A.F. 2010. Analysis 299 of mRNA nuclear export kinetics in mammalian cells by microinjection. J Vis Exp(46). 300 doi: $10.3791 / 2387$.

301 Hatayama, M., Tomizawa, T., Sakai-Kato, K., Bouvagnet, P., Kose, S., Imamoto, N., 302 Yokoyama, S., Utsunomiya-Tate, N., Mikoshiba, K., Kigawa, T., and Aruga, J. 2008. 303 Functional and structural basis of the nuclear localization signal in the ZIC3 zinc finger 304 domain. Hum. Mol. Genet. 17(22): 3459-3473. doi: 10.1093/hmg/ddn239.

305 Hessabi, B., Schmidt, I., and Walther, R. 2000. The homeodomain of Nkx2.2 carries two 306 cooperatively acting nuclear localization signals. Biochem. Biophys. Res. Commun. 307 270(3): 695-700. doi: 10.1006/bbrc.2000.2491.

308 Hovde, S., Abate-Shen, C., and Geiger, J.H. 2001. Crystal structure of the Msx-1 
309 homeodomain/DNA complex. Biochemistry 40(40): 12013-12021.

310 Kamamoto, M., Machida, J., Yamaguchi, S., Kimura, M., Ono, T., Jezewski, P.A., Higashi, Y.,

311 Nakayama, A., Shimozato, K., and Tokita, Y. 2011. Clinical and functional data implicate

312 the $\operatorname{Arg}(151)$ Ser variant of MSX1 in familial hypodontia. Eur J Hum Genet 19(8): 844-850.

313 doi: $10.1038 /$ ejhg.2011.47.

314 Kimura, M., Machida, J., Yamaguchi, S., Shibata, A., Tatematsu, T., Miyachi, H., Jezewski,

315 P.A., Nakayama, A., Higashi, Y., Shimozato, K., and Tokita, Y. 2014. Novel nonsense

316 mutation in MSX1 in familial nonsyndromic oligodontia: subcellular localization and role

317 of homeodomain/MH4. Eur J Oral Sci 122(1): 15-20. doi: 10.1111/eos.12105.

318 Kozlowski, K., and Walter, M.A. 2000. Variation in residual PITX2 activity underlies the

319 phenotypic spectrum of anterior segment developmental disorders. Hum. Mol. Genet.

$320 \quad 9(14): 2131-2139$.

321 Lange, A., Mills, R.E., Lange, C.J., Stewart, M., Devine, S.E., and Corbett, A.H. 2007.

322 Classical nuclear localization signals: Definition, function, and interaction with importin $\alpha$.

323 J. Biol. Chem. 282(8): 5101-5105.

324 Ladam, F., and Sagerstrom, C.G. 2014. Hox regulation of transcription: more complex(es).

325 Developmental dynamics : an official publication of the American Association of 

Anatomists 243(1): 4-15. doi: 10.1002/dvdy.23997.

327 Lee, B.J., Cansizoglu, A.E., Süel, K.E., Louis, T.H., Zhang, Z., and Chook, Y.M. 2006. Rules 328 for Nuclear Localization Sequence Recognition by Karyopherin $\beta 2$. Cell 126(3): 543-558.

329 Medio, M., Yeh, E., Popelut, A., Babajko, S., Berdal, A., and Helms, J.A. 2012.

330 Wnt/beta-catenin signaling and Msx1 promote outgrowth of the maxillary prominences.

$331 \quad$ Front Physiol 3: 375. doi: 10.3389/fphys.2012.00375.

332 Moede, T., Leibiger, B., Pour, H.G., Berggren, P., and Leibiger, I.B. 1999. Identification of a 333 nuclear localization signal, RRMKWKK, in the homeodomain transcription factor PDX-1.

$334 \quad$ FEBS Lett. 461(3): 229-234.

335 Ochman, H., Gerber, A.S., and Hartl, D.L. 1988. Genetic applications of an inverse 336 polymerase chain reaction. Genetics 120(3): 621-623.

337 Pan, G., Qin, B., Liu, N., Scholer, H.R., and Pei, D. 2004. Identification of a nuclear 338 localization signal in OCT4 and generation of a dominant negative mutant by its ablation. J.

339 Biol. Chem. 279(35): 37013-37020. doi: 10.1074/jbc.M405117200.

340 Rave-Harel, N., Miller, N.L., Givens, M.L., and Mellon, P.L. 2005. The Groucho-related gene

341 family regulates the gonadotropin-releasing hormone gene through interaction with the

342 homeodomain proteins MSX1 and OCT1. J. Biol. Chem. 280(35): 30975-30983. doi: 

10.1074/jbc.M502315200.

344 Robbins, J., Dilworth, S.M., Laskey, R.A., and Dingwall, C. 1991. Two interdependent basic

345 domains in nucleoplasmin nuclear targeting sequence: identification of a class of bipartite

346 nuclear targeting sequence. Cell 64(3): 615-623.

347 Sato, S., Morita, S., Iha, M., Mori, Y., Sugawara, S., Kasuga, K., Kojima, I., Ozaki, N.,

348 Muraguchi, H., Okano, K., Iwashita, J., Murata, J., Hosaka, M., and Kobayashi, M. 2013.

349 Intact structure of EGAM1 homeoproteins and basic amino acid residues in the common

350 homeodomain of EGAM1 and EGAM1C contribute to their nuclear localization in mouse

351 embryonic stem cells. J. Biosci. Bioeng. 116(2): 141-146. doi:

$352 \quad 10.1016 / j . j b i o s c .2013 .02 .007$.

353 Satokata, I., and Maas, R. 1994. Msx1 deficient mice exhibit cleft palate and abnormalities of

354 craniofacial and tooth development. Nat. Genet. 6(4): 348-356.

355 Schneider, C.A., Rasband, W.S., and Eliceiri, K.W. 2012. NIH Image to ImageJ: 25 years of

356 image analysis. Nat. Methods 9(7): 671-675.

357 Sock, E., Enderich, J., Rosenfeld, M.G., and Wegner, M. 1996. Identification of the nuclear

358 localization signal of the POU domain protein Tst-1/Oct6. J. Biol. Chem. 271(29):

$359 \quad 17512-17518$. 
360 Tokita, Y., Keino, H., Matsui, F., Aono, S., Ishiguro, H., Higashiyama, S., and Oohira, A. 2001.

361 Regulation of neuregulin expression in the injured rat brain and cultured astrocytes. J.

$362 \quad$ Neurosci. 21(4): 1257-1264.

363 Wang, Y., Kong, H., Mues, G., and D'Souza, R. 2011. Msx1 mutations: how do they cause

364 tooth agenesis? J. Dent. Res. 90(3): 311-316. doi: 10.1177/0022034510387430.

365 Wong, S.W., Liu, H.C., Han, D., Chang, H.G., Zhao, H.S., Wang, Y.X., and Feng, H.L. 2014.

366 A novel non-stop mutation in MSX1 causing autosomal dominant non-syndromic

367 oligodontia. Mutagenesis. doi: 10.1093/mutage/geu019.

368 Ye, W., Lin, W., Tartakoff, A.M., and Tao, T. 2011. Karyopherins in nuclear transport of

369 homeodomain proteins during development. Biochim. Biophys. Acta 1813(9): 1654-1662.

$370 \quad$ doi: 10.1016/j.bbamcr.2011.01.013.

371 Zhang, H., Catron, K.M., and Abate-Shen, C. 1996. A role for the Msx-1 homeodomain in

372 transcriptional regulation: Residues in the N-terminal arm mediate TATA binding protein

373 interaction and transcriptional repression. Proceedings of the National Academy of

374 Sciences of the United States of America 93(5): 1764-1769.

375 Zhang, H., Hu, G., Wang, H., Sciavolino, P., Iler, N., Shen, M.M., and Abate-Shen, C. 1997.

376 Heterodimerization of Msx and Dlx homeoproteins results in functional antagonism. Mol. 
377 Cell. Biol. 17(5): 2920-2932.

378

379 
380 Table. 1. NLS varies with the individual by the classification and location.

381 


\section{Figure captions}

383 Figure 1. The nuclear localization of MSX1 depends on its HD. (A) Schematic diagrams of

384 FLAG tagged wild-type MSX1, its deletion mutants, $\triangle \mathrm{HD}$ and HD. The amino acid

385 sequences of the putative NLS predicted by a web-based tool, NLStradamus

386 (http://www.moseslab.csb.utoronto.ca/NLStradamus/) are shown. (B) The

387 immunolocalization of FLAG tagged wild-type MSX1 and its deletion mutants in HEK293

388 cells were detected using the mouse anti-FLAG antibody and Cy3-conjugated anti-mouse IgG

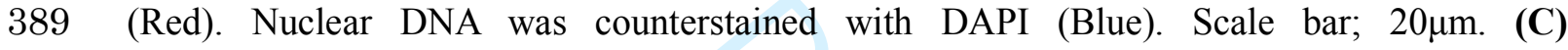

390 Photographs of randomly selected fields were taken under the same conditions, and all cells

391 within each field were scored according to signal intensity distribution in the nucleus and

392 cytoplasm (see materials and methods). The percentage of nuclear fluorescence represents the

393 mean \pm SEM from two experiments in which 50 cells were scored per experiment. The

394 p-values compare the percentage of nuclear fluorescence in the control versus each HD

395 protein mutants according to the Welch's $\mathrm{t}$ test. *** $p>0.001$ versus each fluorescence.

396

397 Figure 2. Mapping of the NLS motifs in the MSX1 protein. (A) Schematic diagram of

398 EGFP tagged MSX1-HD and its deletion mutants. Deletion mutants of the EGFP MSX-HD 
399 fusion protein $\left(\mathrm{HDN}^{\prime}{ }_{161-191}, \mathrm{HDC}^{\prime}{ }_{192-228,} \Delta \mathrm{NLS1}\right.$ and $\left.\Delta \mathrm{NLS} 2\right)$ were constructed. (B)

400 Subcellular localization of these mutant fusion proteins detected using anti-GFP antibody and

401 Alexa488-labeled secondary antibodies (Green). Nuclear DNA was counterstained with DAPI

402 (Blue). Scale bar; $20 \mu \mathrm{m}$. (C) The percentage of nuclear fluorescence of the chimeric proteins.

403 The values represent the mean \pm SEM from two experiments in which 50 cells were scored

404 per experiment. The p-values compare the percentage of nuclear fluorescence in the control

405 versus each HD protein mutants according to the Welch's $\mathrm{t}$ test. ${ }^{* * *} p>0.001$ versus

406 EGFP-HD and \# $p>0.001$ versus EGFP.

407

408 Figure 3. Cooperative effect of the isolated NLS1 and 2 motifs. (A) Schematic diagram of

409 EGFP fusion proteins of an entire HD. NLS1 and NLS1+2 were constructed. (B) Subcellular

410 localization of mutant fusion proteins. The subcellular localization of EGFP-fusion proteins

411 was detected using anti-GFP antibody and Alexa488-labeled secondary antibodies (Green).

412 Nuclear DNA was counterstained with DAPI (Blue). Scale bar; 20 $\mu \mathrm{m}$. (C) The percentage of

413 nuclear fluorescence of the chimeric proteins. The values represent the mean \pm SEM from two

414 experiments in which 50 cells were scored per experiment. The p-values compare the

415 percentage of nuclear fluorescence in the control versus each HD protein mutants according 
416 to the Welch's t test. ${ }^{* * *} p>0.001$ versus EGFP-HD and $\# p>0.001$ versus EGFP. (D)

417 Western blot analysis for EGFP was performed on cytosolic and nuclear extracts prepared 418 from HEK293 cells transfected transiently with EGFP EGFP-HD or EGFP-NLS1+2 (Upper

419 panel). Histone H3 and tubulin were used as loading controls (lower panels). Nuclear:

420 Nuclear fraction, Cytosolic: Cytosolic fraction.

421

422 Figure 4. Characterization of the tandem two NLS motifs in MSX1. (A) Schematic

423 diagram of the mutants of the EGFP NLS1+2 fusion protein (mNLS1N', mNLS1C', mNLS1,

424 mNLS2 and mNLS1+2) were constructed. (B) Subcellular localization of mutant fusion

425 proteins. The subcellular localization of EGFP-fusion proteins was detected using anti-GFP

426 antibody and Alexa488-labeled secondary antibodies (Green). Nuclear DNA was

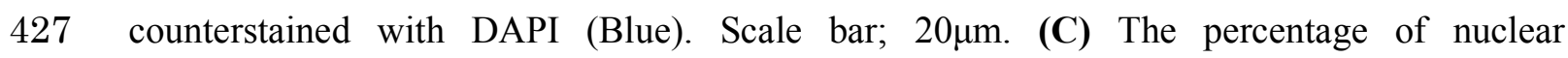

428 fluorescence of the chimeric proteins. The values represent the mean \pm SEM from two

429 experiments in which 50 cells were scored per experiment. The p-values compare the

430 percentage of nuclear fluorescence in the control versus each HD protein mutants according

431 to the Welch's t test. $* * * p>0.001$ versus EGFP-NLS1+2 and \# $p>0.001$ versus EGFP. 


\section{Supporting Information}

434 S1 Table. Oligonucleotide primers for mutagenesis of MSX1 HD 
A
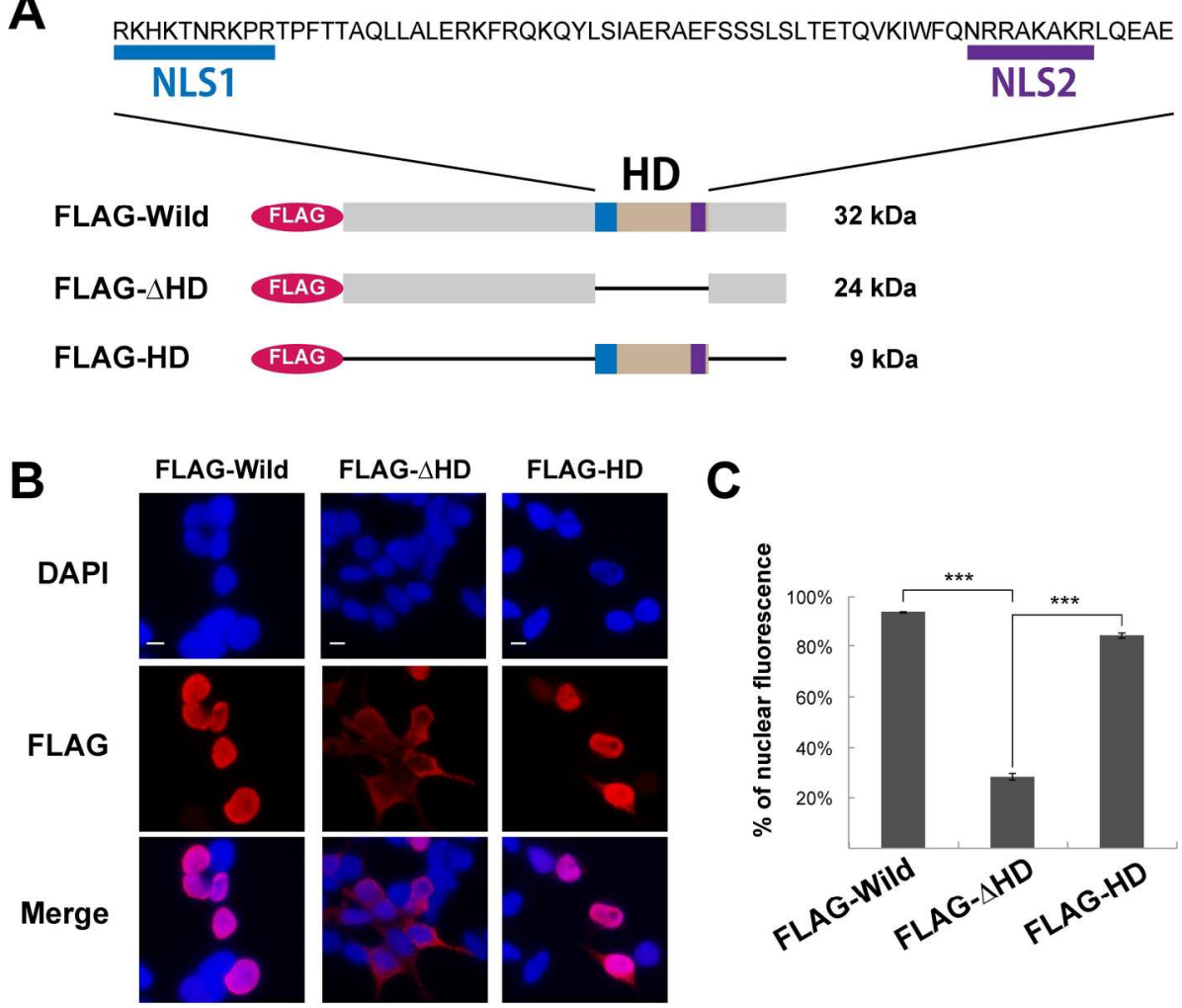

Figure 1. The nuclear localization of MSX1 depends on its HD. (A) Schematic diagrams of FLAG tagged wildtype MSX1, its deletion mutants, $\triangle H D$ and HD. The amino acid sequences of the putative NLS predicted by a web-based tool, NLStradamus (http://www.moseslab.csb.utoronto.ca/NLStradamus/) are shown. (B) The immunolocalization of FLAG tagged wild-type MSX1 and its deletion mutants in HEK293 cells were detected using the mouse anti-FLAG antibody and Cy3-conjugated anti-mouse IgG (Red). Nuclear DNA was counterstained with DAPI (Blue). Scale bar; $20 \mu \mathrm{m}$. (C) Photographs of randomly selected fields were taken under the same conditions, and all cells within each field were scored according to signal intensity distribution in the nucleus and cytoplasm (see materials and methods). The percentage of nuclear fluorescence represents the mean \pm SEM from two experiments in which 50 cells were scored per experiment. The $p$-values compare the percentage of nuclear fluorescence in the control versus each HD protein mutants according to the Welch's t test. $* * * \mathrm{p}>0.001$ versus each fluorescence.

$180 \times 154 \mathrm{~mm}(300 \times 300 \mathrm{DPI})$ 


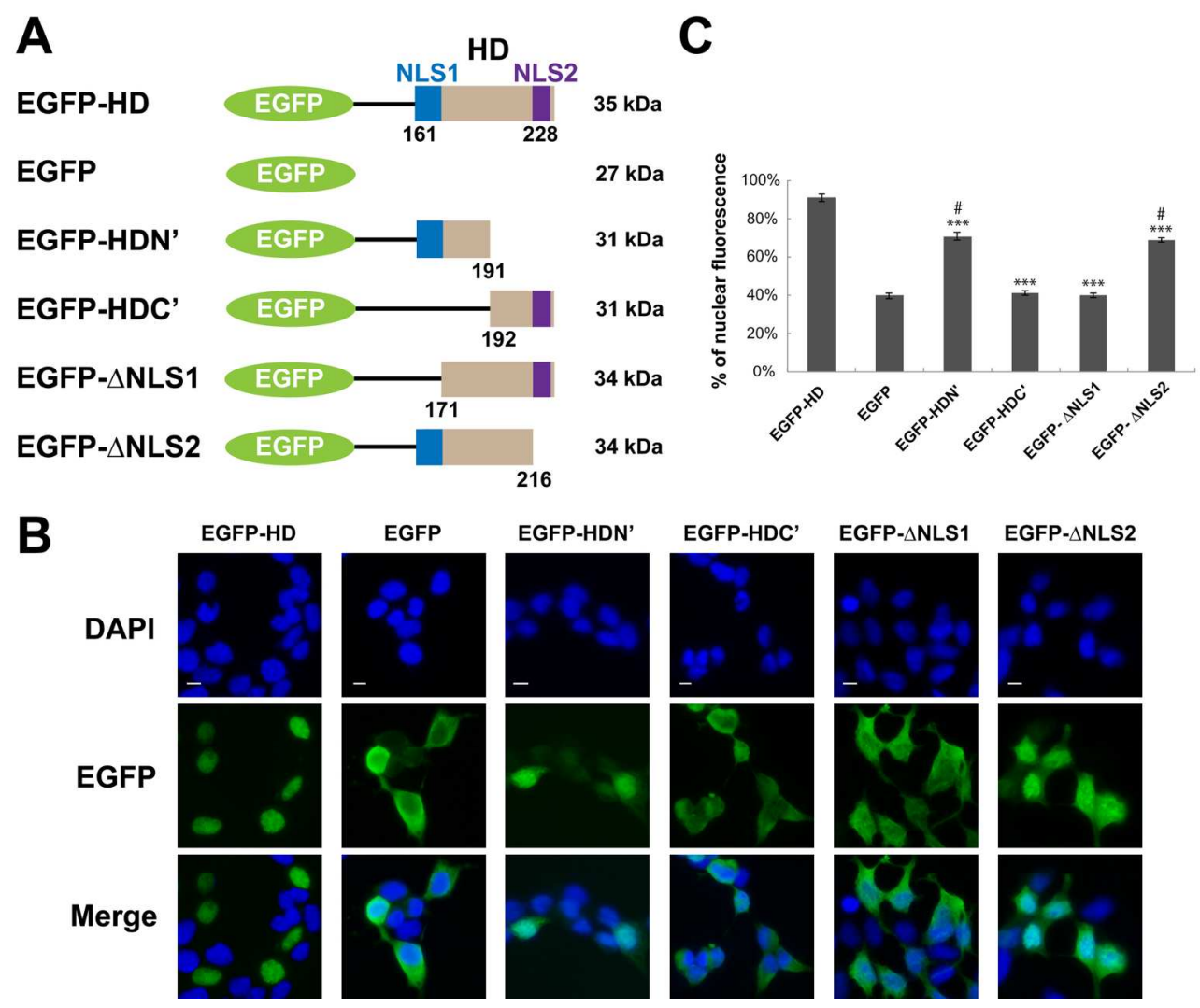

Figure 2. Mapping of the NLS motifs in the MSX1 protein. (A) Schematic diagram of EGFP tagged MSX1-HD and its deletion mutants. Deletion mutants of the EGFP MSX-HD fusion protein (HDN'161-191, HDC'192228, $\triangle$ NLS1 and $\triangle$ NLS2) were constructed. (B) Subcellular localization of these mutant fusion proteins detected using anti-GFP antibody and Alexa488-labeled secondary antibodies (Green). Nuclear DNA was counterstained with DAPI (Blue). Scale bar; $20 \mu \mathrm{m}$. (C) The percentage of nuclear fluorescence of the chimeric proteins. The values represent the mean \pm SEM from two experiments in which 50 cells were scored per experiment. The $p$-values compare the percentage of nuclear fluorescence in the control versus each HD protein mutants according to the Welch's t test. *** $\mathrm{p}>0.001$ versus EGFP-HD and \# $\mathrm{p}>0.001$ versus EGFP.

$167 \times 139 \mathrm{~mm}(300 \times 300 \mathrm{DPI})$ 


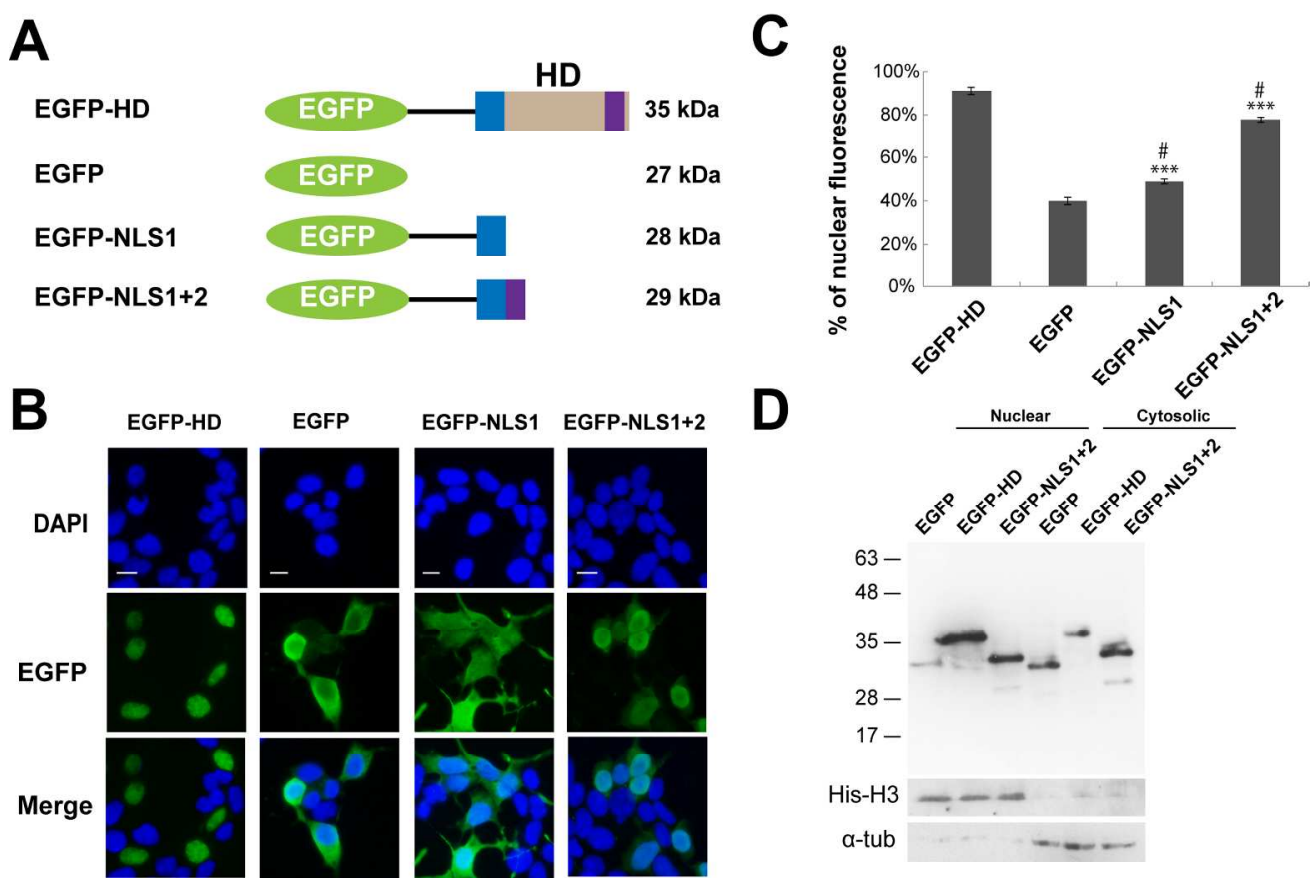

Figure 3. Cooperative effect of the isolated NLS1 and 2 motifs. (A) Schematic diagram of EGFP fusion proteins of an entire HD. NLS1 and NLS1+2 were constructed. (B) Subcellular localization of mutant fusion proteins. The subcellular localization of EGFP-fusion proteins was detected using anti-GFP antibody and Alexa488-labeled secondary antibodies (Green). Nuclear DNA was counterstained with DAPI (Blue). Scale bar; $20 \mu \mathrm{m}$. (C) The percentage of nuclear fluorescence of the chimeric proteins. The values represent the mean \pm SEM from two experiments in which 50 cells were scored per experiment. The $p$-values compare the percentage of nuclear fluorescence in the control versus each HD protein mutants according to the Welch's $t$ test. $* * * p>0.001$ versus EGFP-HD and \# $p>0.001$ versus EGFP. (D) Western blot analysis for EGFP was performed on cytosolic and nuclear extracts prepared from HEK293 cells transfected transiently with EGFP EGFP-HD or EGFP-NLS1+2 (Upper panel). Histone H3 and tubulin were used as loading controls (lower panels). Nuclear: Nuclear fraction, Cytosolic: Cytosolic fraction.

$195 \times 130 \mathrm{~mm}(300 \times 300 \mathrm{DPI})$ 
A

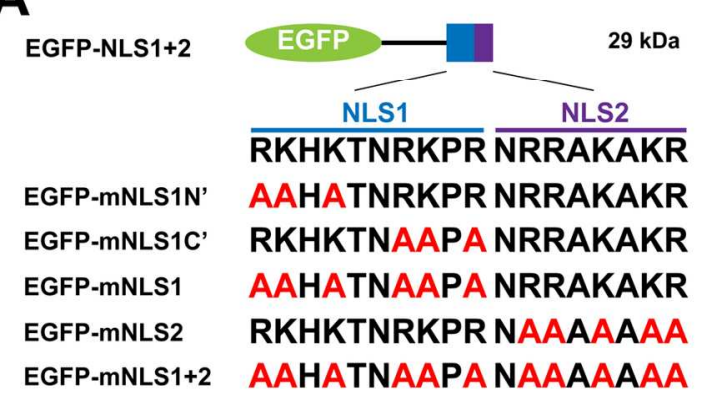

C

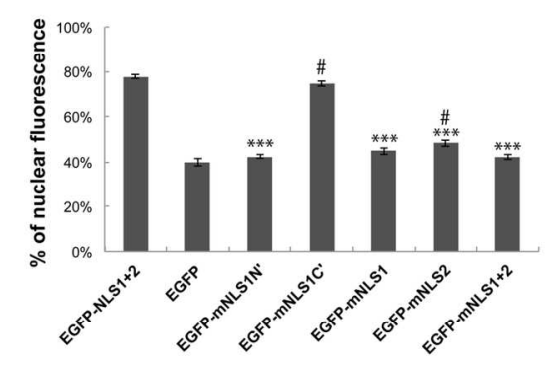

B

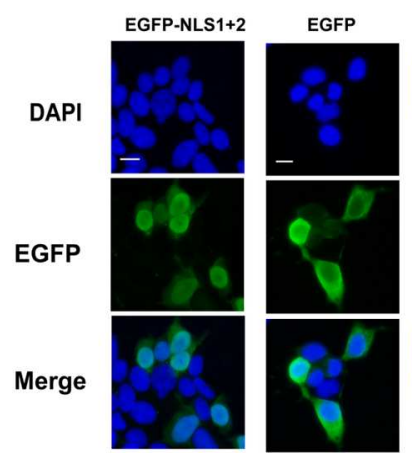

EGFP-mNLS1N' EGFP-mNLS1C
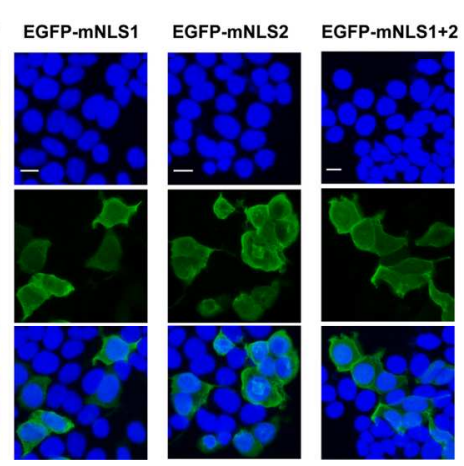

Figure 4. Characterization of the tandem two NLS motifs in MSX1. (A) Schematic diagram of the mutants of the EGFP NLS1+2 fusion protein (mNLS1N', mNLS1C', mNLS1, mNLS2 and mNLS1+2) were constructed. (B) Subcellular localization of mutant fusion proteins. The subcellular localization of EGFP-fusion proteins was detected using anti-GFP antibody and Alexa488-labeled secondary antibodies (Green). Nuclear DNA was counterstained with DAPI (Blue). Scale bar; $20 \mu \mathrm{m}$. (C) The percentage of nuclear fluorescence of the chimeric proteins. The values represent the mean \pm SEM from two experiments in which 50 cells were scored per experiment. The $p$-values compare the percentage of nuclear fluorescence in the control versus each HD protein mutants according to the Welch's t test. $* * * \mathrm{p}>0.001$ versus EGFP-NLS1+2 and \# $\mathrm{p}>$ 0.001 versus EGFP. 
1 Table. 1. NLS varies with the individual by the classification and location.

\begin{tabular}{ccccc}
\multirow{2}{*}{ Protein } & Classification & \multicolumn{2}{c}{ Location of NLS } & Reference \\
\hline Tst-1/Oct6 & monopartite & GRKRKKRT & C-terminus of HD & Sock et al. 1996 \\
PITX2 & monopartite & & RVRVWFKNRRAKWRKR & Kozlowski and Walter 2000 \\
PDX1 & monopartite & & RRMKWKK & Moede et al. 1999 \\
Dlx3 & bipartite & GKPKKVRK..14A.a..RRFK & & Bryan and Morasso 2000 \\
Nanog & two NLS & KQKTR & RMKSKRWQ & Do et al. 2007 \\
Nkx2.2 & two NLS & KKRKRR & RYKMKRAR & Hessabi et al. 2000 \\
TTF-1 & two NLS & PRRKRRV & WFQ & Christophe-Hobertus et al. 1999 \\
MSX1 & two NLS & RKHKTNRKPR & RRAKAKR & This study \\
\hline
\end{tabular}

2

HD: Homeodomain, NLS: Nuclear localization signal 


\begin{tabular}{|c|c|c|c|c|}
\hline \multirow{2}{*}{ Name/ tamplate } & & \multicolumn{2}{|c|}{ Primer } & \multirow{2}{*}{ PCR method } \\
\hline & & 5 ' terminus & 3 'terminus & \\
\hline \multicolumn{5}{|l|}{$\overline{\mathrm{pcDNA} 3}$} \\
\hline entire HD & & AGATCTCGCAAACACAAGACGAACCG & GAGACTACAAGAGGCAGAGTAGGTCCAG & Conventional \\
\hline HDN' & & CAGGTACTGCTTCTGGCGGAACT & TAGCTCGAGCATGCATCTAGAGG & Inverse \\
\hline $\mathrm{HDC}^{\prime}$ & & ATGGACTACAAGGACGACGACGACAAG & TCCATCGCCGAGCGCGCGGAGTT & Inverse \\
\hline \multicolumn{5}{|l|}{ EGFPC1 } \\
\hline $\mathrm{HDN}^{\prime}$ & & AGATCTCGCAAACACAAGACGAACCG & CGCCAGAAGCAGTACCTGTAGGTCCAG & Conventional \\
\hline $\mathrm{HDC}^{\prime}$ & & AGATCTTCCATCGCCGAGCGCGC & GAGACTACAAGAGGCAGAGTAGGTCCAG & Conventional \\
\hline$\triangle \mathrm{NLS1}$ & & CGAGCTGTACAAGTCCGGACTCAGATCT & ACGCCCTTCACCACCGCGCAGCTG & Inverse \\
\hline$\triangle \mathrm{NLS} 2$ & & ACGCAGGTGAAGATATGGTTCCAGAAC & CAAGAGGCAGAGTAGGTCCAGATCACT & Inverse \\
\hline $\mathrm{NLS} 1+2$ & & CAAACACAAGACGAACCGTAAGCCGCGG & CGCCGCGCCAAGGCAAAGAGACTACAA & Inverse \\
\hline \multirow[t]{2}{*}{ mNLS1N' } & 1st & GATCCAGATCTGCCGC ACACAAGACGA & & Mega \\
\hline & 2nd & AGATCTGCCGCACACGCGACGA ACCGT & & Mega \\
\hline \multirow[t]{2}{*}{ mNLS1C' } & 1 st & CCGTAAGCCGGCGAACCGCCGCGCCA & & Mega \\
\hline & 2nd & ACAAGACGAACGCTGCGCCGGCGAAC & & Mega \\
\hline \multirow[t]{3}{*}{ mNLS2 } & 1 st & CGCCAAGGCAGCGGCACTACAAGAGG & & Mega \\
\hline & 2 nd & AACCGCCGCGCCGCGGCAGCGGCACT & & Mega \\
\hline & 3 rd & TAAGCCGCGGAACGCCGCCGCCGCGGCA & & Mega \\
\hline NLS1 & & ACGCGACGAACGCTGCGCCGGCGAAC & & Mega \\
\hline $\mathrm{NLS} 1+2$ & & TGCGCCGGCGAACGCCGCCGCCGCGGCA & & Mega \\
\hline mega primer 5'terminus & & ATTGATAAGTACTTGAAATCCAGCAAGT & & - \\
\hline mega primer 3 'temminus & & TCAGTTATCTAGATCCGGTGGATCCCG & & - \\
\hline
\end{tabular}




\section{9:enago Author First, Quality First 英文校正・校閲-エナゴ}

Biochemistry and Cell Biology

\section{ulatus}

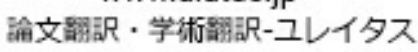

\title{
CERTIFICATE OF EDITING
}

This is to certify that the paper titled Identification of Nuclear Localization Signals in the Human Homeoprotein, MSX1 commissioned to us by Akio Shibata (大垣市 民病院) has been edited for English language, grammar, punctuation, and spelling by Enago, the editing brand of Crimson Interactive Pvt. Ltd under Normal Editing

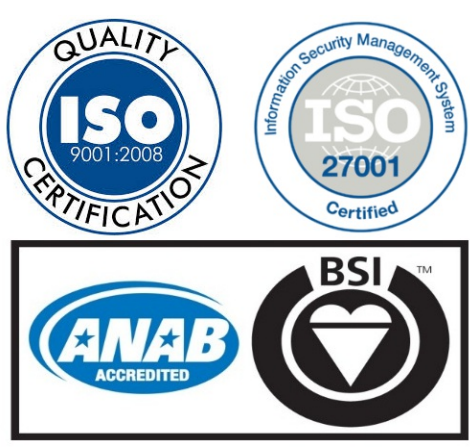

\author{
Issued by: 233 \\ Enago, Grimson Interactive Pvt. Ltd. \\ 1001, Techniplex - II, S. V. Road, \\ Goregaon (W), Mumbai 400062, India \\ Phone: 03-5050-5374 \\ Fax: 03-4496-4934
}

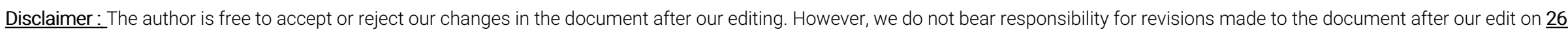
Oct 2017.

$\begin{array}{llll}\text { Global } & \text { www.enago.com, www.ulatus.com, www.voxtab.com } & \text { Germany www.enago.de } \\ \text { Japan } & \text { www.enago.jp, www.ulatus.jp, www.voxtab.jp } & \text { Russia } & \text { www.enago.ru } \\ \text { Taiwan } & \text { www.enago.tw } & \text { Arabic } & \text { www.enago.ae } \\ \text { China } & \text { www.enago.cn } & \text { Turkey } & \text { www.enago.com.tr } \\ \text { Brazil } & \text { www.enago.com.br } & \text { S. Korea } & \text { www.enago.co.kr }\end{array}$

Society for Scholarly Publishing

\section{ISM TE}

\section{About Crimson:}

Crimson Interactive Inc. provides English language editing, transcription, and translation services to individuals and corporate customers worldwide.

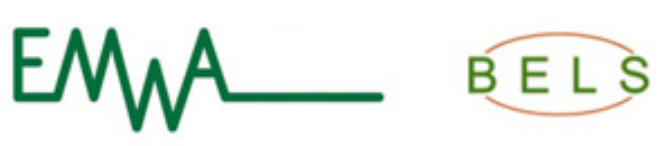

